\title{
Weak and strong singularities problems to Liénard equation
}

Yun Xin ${ }^{1}$ and Guixin $\mathrm{Hu}^{2 *}$

\section{${ }^{*}$ Correspondence:}

hgx_1982@126.com

${ }^{2}$ School of Mathematics and

Information Science, Henan

Polytechnic University, Jiaozuo 454000, China

Full list of author information is available at the end of the article

\begin{abstract}
This paper is devoted to an investigation of the existence of a positive periodic solution for the following singular Liénard equation:

$$
x^{\prime \prime}+f(x(t)) x^{\prime}(t)+a(t) x=\frac{b(t)}{x^{\alpha}}+e(t)
$$

where the external force $e(t)$ may change sign, $\alpha$ is a constant and $\alpha>0$. The novelty of the present article is that for the first time we show that weak and strong singularities enables the achievement of a new existence criterion of positive periodic solution through an application of the Manásevich-Mawhin continuation theorem. Recent results in the literature are generalized and significantly improved, and we give the existence interval of periodic solution of this equation. At last, two examples and numerical solution (phase portraits and time portraits of periodic solutions of the example) are given to show applications of the theorem.
\end{abstract}

MSC: 34B16; 34B18; 34C25

Keywords: Weak and strong singularities; Liénard equation; Positive periodic solution

\section{Introduction}

The main purpose of this paper is to consider the existence of a periodic solution for the Liénard equation with weak and strong singularities of repulsive type,

$$
x^{\prime \prime}+f(x(t)) x^{\prime}(t)+a(t) x=\frac{b(t)}{x^{\alpha}}+e(t),
$$

where $a, b \in C(\mathbb{R},(0,+\infty))$ are $\omega$-periodic functions, $f \in C(\mathbb{R}, \mathbb{R})$, the external force $e \in$ $C(\mathbb{R}, \mathbb{R})$ is an $\omega$-periodic function. Moreover, note that when $f(x(t)) \equiv 0$, Eq. (1.1) becomes

$$
x^{\prime \prime}+a(t) x=\frac{b(t)}{x^{\alpha}}+e(t) .
$$

(c) The Author(s) 2020. This article is licensed under a Creative Commons Attribution 4.0 International License, which permits use, sharing, adaptation, distribution and reproduction in any medium or format, as long as you give appropriate credit to the original author(s) and the source, provide a link to the Creative Commons licence, and indicate if changes were made. The images or other third party material in this article are included in the article's Creative Commons licence, unless indicated otherwise in a credit line to the material. If material is not included in the article's Creative Commons licence and your intended use is not permitted by statutory regulation or exceeds the permitted use, you will need to obtain permission directly from the copyright holder. To view a copy of this licence, visit http://creativecommons.org/licenses/by/4.0/. 
In 1987, Lazer and Solimini [1] investigated the following second-order differential equation with singularity of repulsive type:

$$
x^{\prime \prime}=\frac{1}{x^{\alpha}}+h(t)
$$

and obtained the result that if the external force $h(t)$ was continuous and $\omega$-periodic, then for all $\alpha>0$ a positive periodic solution existed if and only if the external force $h(t)$ has a positive mean value. We say the equation to obey the strong force condition if $\alpha \geq 1$ and the weak force condition if $0<\alpha<1$.

Lazer and Solimini's work has attracted the attention of many scholars in singular equations. More recently, the Poincaré-Birkhoff twist theorem [2-4], Schauder's fixed point theorem [5-8], the Leray-Schauder alternative principle [9-11], coincidence degree theory [12-15],the Krasnoselskii fixed point theorem in cones [16, 17] and Leray-Schauder degree theory $[18,19]$ have been employed to discuss the existence of a positive periodic solution of singular equations.

Among these papers, there have been published some results on Eq. (1.2) (see [5, 6, 8, 10, 17]). Chu et al. [10] in 2007 discussed the existence of a positive periodic solution for Eq. (1.2) if the external force $e(t) \geq 0$ and $\|a\|:=\max _{t \in[0, \omega]}|a(t)|<\frac{\pi^{2}}{\omega^{2}}$. Their results were based on a nonlinear alternative principle of Leray-Schauder and are applicable to the case of a strong singularity and the case of a weak singularity. Afterwards, Torres [8] proved Eq. (1.2) in the cases of weak and strong singularities had at least one positive periodic solution if the external force $e(t)>0$ and $\|a\|<\frac{\pi^{2}}{\omega^{2}}$. Moreover, the author obtained the result that there was one positive periodic solution for Eq. (1.2) in the case of a weak singularity if one of the following conditions holds:

(i) $e(t) \equiv 0$ and $\|a\|<\frac{\pi^{2}}{\omega^{2}}$; or (ii) $e(t)<0$ and $\|a\|<\frac{\pi^{2}}{\omega^{2}}$.

Wang [17] in 2010 improved the above result and presented a new assumption, which is weaker than the singular condition in [8]. The author obtained the result that Eq. (1.2) in the cases that weak and strong singularities have at least one positive periodic solution if and only if one of the following conditions holds:

(i) $e(t) \geq 0$ and $\|a\|<\frac{\pi^{2}}{\omega^{2}}$; or (ii) $e(t)<0$ and $\|a\|<\frac{\pi^{2}}{\omega^{2}}$.

The proof of their results was based on the Krasnoselskii fixed point theorem in a cone. All the aforementioned results are related to Eq. (1.2) with the external force $e(t)$ not changing sign. Naturally, a new question arises: how may Eq. (1.1) with weak and strong singularities work on the external force $e(t)$ changing sign? In this paper, we fill the gap and provide sufficient conditions for the existence of a positive periodic solution for Eq. (1.1) with weak and strong singularities, where the external force $e(t)$ may change sign, $\alpha$ is a constant and $\alpha>0$. By applications of the Manásevich-Mawhin continuation theorem [20, Theorem 3.1], we obtain the following conclusion.

Theorem 1.1 Assume that the following conditions are satisfied:

$\left(H_{1}\right) \int_{0}^{\omega} e(t) d t=0$;

$\left(H_{2}\right) \quad\|a\|<\frac{\pi^{2}}{\omega^{2}}$;

$\left(H_{3}\right)$

$$
\begin{aligned}
& \sigma_{*}>\left(\frac{\omega^{\frac{1}{2}}}{2}\left(\frac{\pi^{2} \omega\left(\|a\|\left(\sigma^{*}\right)^{\frac{2}{1+\alpha}}+\|e\|\left(\sigma^{*}\right)^{\frac{1}{1+\alpha}}\right)}{\pi^{2}-\|a\| \omega^{2}}\right)^{\frac{1}{2}}+\frac{\pi \omega^{2}\left(2\|a\|\left(\sigma^{*}\right)^{\frac{1}{1+\alpha}}+\|e\|\right)}{2\left(\pi^{2}-\|a\| \omega^{2}\right)}\right)^{1+\alpha}, \\
& \text { where } \sigma^{*}:=\frac{b^{*}}{a_{*}}, \sigma_{*}:=\frac{b_{*}}{a^{*}}, b^{*}:=\max _{t \in[0, \omega]} b(t), b_{*}:=\min _{t \in[0, \omega]} b(t) .
\end{aligned}
$$


Then Eq. (1.1) has at least one positive periodic solution $x$ with

$$
\begin{array}{r}
x \in\left(\left(\sigma_{*}\right)^{\frac{1}{1+\alpha}}-\frac{\omega^{\frac{1}{2}}}{2}\left(\frac{\pi^{2} \omega\left(\|a\|\left(\sigma^{*}\right)^{\frac{2}{1+\alpha}}+\|e\|\left(\sigma^{*}\right)^{\frac{1}{1+\alpha}}\right)}{\pi^{2}-\|a\| \omega^{2}}\right)^{\frac{1}{2}}-\frac{\pi \omega^{2}\left(2\|a\|\left(\sigma^{*}\right)^{\frac{1}{1+\alpha}}+\|e\|\right)}{2\left(\pi^{2}-\|a\| \omega^{2}\right)},\right. \\
\left.\left(\sigma^{*}\right)^{\frac{1}{1+\alpha}}+\frac{\omega^{\frac{1}{2}}}{2}\left(\frac{\pi^{2} \omega\left(\|a\|\left(\sigma^{*}\right)^{\frac{2}{1+\alpha}}+\|e\|\left(\sigma^{*}\right)^{\frac{1}{1+\alpha}}\right)}{\pi^{2}-\|a\| \omega^{2}}\right)^{\frac{1}{2}}+\frac{\pi \omega^{2}\left(2\|a\|\left(\sigma^{*}\right)^{\frac{1}{1+\alpha}}+\|e\|\right)}{2\left(\pi^{2}-\|a\| \omega^{2}\right)}\right) .
\end{array}
$$

Remark 1.1 The techniques used are quite different from that in $[5,8,10,17]$ and our results are more general than those in $[5,8,10,17]$ in two aspects. We first obtain the existence of a positive periodic solution for equation (1.1) with weak and strong singularities if the external force $e(t)$ may change sign. Secondly, we give the existence interval of positive periodic solution of Eq. (1.1).

In the following, we consider the existence of a periodic solution for Eq. (1.1) without the external force $e(t)$.

Corollary 1.1 Assume that $\left(H_{2}\right)$ holds. Furthermore, suppose the following conditions are satisfied:

$$
\begin{aligned}
& \left(H_{1}^{\prime}\right) \quad e(t)=0 ; \\
& \left(H_{3}^{\prime}\right) \quad \sigma_{*}>\left(\frac{\omega^{\frac{1}{2}}}{2}\left(\frac{\pi^{2} \omega\|a\|\left(\sigma^{*}\right) \frac{2}{1+\alpha}}{\pi^{2}-\|a\| \omega^{2}}\right)^{\frac{1}{2}}+\frac{\pi \omega^{2}\|a\|\left(\sigma^{*}\right) \frac{1}{1+\alpha}}{\left(\pi^{2}-\|a\| \omega^{2}\right)}\right)^{1+\alpha} .
\end{aligned}
$$

Then Eq. (1.1) has at least one positive periodic solution $x$ with

$$
\begin{array}{r}
x \in\left(\left(\sigma_{*}\right)^{\frac{1}{1+\alpha}}-\frac{\omega^{\frac{1}{2}}}{2}\left(\frac{\pi^{2} \omega\|a\|\left(\sigma^{*}\right)^{\frac{2}{1+\alpha}}}{\pi^{2}-\|a\| \omega^{2}}\right)^{\frac{1}{2}}-\frac{\pi \omega^{2}\|a\|\left(\sigma^{*}\right)^{\frac{1}{1+\alpha}}}{\left(\pi^{2}-\|a\| \omega^{2}\right)},\right. \\
\left.\left(\sigma^{*}\right)^{\frac{1}{1+\alpha}}+\frac{\omega^{\frac{1}{2}}}{2}\left(\frac{\pi^{2} \omega\|a\|\left(\sigma^{*}\right)^{\frac{2}{1+\alpha}}}{\pi^{2}-\|a\| \omega^{2}}\right)^{\frac{1}{2}}+\frac{\pi \omega^{2}\|a\|\left(\sigma^{*}\right)^{\frac{1}{1+\alpha}}}{\left(\pi^{2}-\|a\| \omega^{2}\right)}\right) .
\end{array}
$$

Obviously, the condition $\left(H_{3}\right)$ (or $\left.\left(H_{3}^{\prime}\right)\right)$ is hard restrictive for the existence of a positive periodic solution to Eq. (1.1). In the following, we study the existence of a positive periodic solution for Eq. (1.1) with strong singularity (i.e. $\alpha \geq 1)$ if conditions $\left(H_{1}\right)$ and $\left(H_{2}\right)$ are satisfied.

Theorem 1.2 Assume that conditions $\left(H_{1}\right)$ and $\left(H_{2}\right)$ hold. Furthermore, suppose the following condition is satisfied:

$\left(H_{4}\right) \quad b(t) \equiv b$ and $b$ is a positive constant.

Then Eq. (1.1) has at least one positive periodic solution if $\alpha \geq 1$.

\section{Proof of theorems}

We first recall the Sobolev inequality recently proved in [21, P. 357] and the topological degree theorem by Mawhin [20].

Lemma 2.1 (Sobolev inequality; see [21]) Let $u \in W^{1, p}(\mathbb{R})$ and $u(0)=u(\omega)=0$. Then we have

$$
S(\sigma) \omega^{-1-2 / \sigma}\|x\|_{\sigma}^{2} \leq\left\|x^{\prime}\right\|_{2}^{2}
$$


where $S(\sigma)$ is the Sobolev constant, $\sigma$ is a positive constant and $\sigma>1, S(\sigma)=\frac{4\left(1+\frac{\sigma}{2}\right)^{2} B^{2}\left(\frac{1}{\sigma}, \frac{1}{2}\right)}{\sigma^{2}\left(1+\frac{1}{\sigma}\right)}$ and $B(\cdot, \cdot)$ is the Beta function.

Remark 2.1 When $\sigma=2$, we get $S(2)=\pi^{2}$.

Next, we investigate a family of (1.1) as follows:

$$
x^{\prime \prime}(t)+\lambda\left(f(x(t)) x^{\prime}(t)+a(t) x(t)-\frac{b(t)}{x^{\alpha}(t)}\right)=\lambda e(t), \quad \lambda \in(0,1] .
$$

Using [20, Theorem 3.1], we obtain the following conclusion.

Lemma 2.2 Assume that there exist positive constants $E_{1}, E_{2}, E_{3}$ and $E_{1}<E_{2}$ such that the following conditions hold:

(1) Each possible periodic solution $x$ to Eq. (2.2) such that $E_{1}<x(t)<E_{2}, \forall t \in[0, \omega]$ and $\left\|x^{\prime}\right\|<E_{3}$.

(2) Each possible solution $C$ to the equation

$$
\int_{0}^{\omega}\left(a(t) C-\frac{b(t)}{C^{\alpha}}\right) d t=0
$$

satisfies $C \in\left(E_{1}, E_{2}\right)$.

(3) We have

$$
\int_{0}^{\omega}\left(a(t) E_{1}-\frac{b(t)}{E_{1}^{\alpha}}\right) d t \cdot \int_{0}^{\omega}\left(a(t) E_{2}-\frac{b(t)}{E_{2}^{\alpha}}\right) d t<0 .
$$

Then Eq. (1.1) has at least one positive periodic solution.

We investigate the existence of a periodic solution for Eq. (1.1) with weak and strong singularities.

Proof of Theorem 1.1 Integrating Eq. (2.2) from 0 to $\omega$, we get

$$
\int_{0}^{\omega}\left(a(t) x(t)-\frac{b(t)}{x^{\alpha}(t)}\right) d t=0
$$

In view of the mean value theorem of integrals, we know that there exists a point $\xi \in(0, \omega)$ such that

$$
a(\xi) x(\xi)=\frac{b(\xi)}{x^{\alpha}(\xi)}
$$

since $\int_{0}^{\omega} x^{\prime \prime}(t) d t=0$ and $\int_{0}^{\omega} e(t) d t=0$ from condition $\left(H_{1}\right)$. Furthermore, we deduce

$$
\left(\sigma_{*}\right)^{\frac{1}{1+\alpha}} \leq x(\xi) \leq\left(\sigma^{*}\right)^{\frac{1}{1+\alpha}}
$$


Multiplying both sides of Eq. (2.2) by $x(t)$ and integrating on the interval $[0, \omega]$, we obtain

$$
\begin{aligned}
& \int_{0}^{\omega} x^{\prime \prime}(t) x(t) d t+\lambda \int_{0}^{\omega} f(x(t)) x(t) x^{\prime}(t) d t+\lambda \int_{0}^{\omega} a(t)|x(t)|^{2} d t \\
& \quad=\lambda \int_{0}^{\omega} b(t) x^{1-\alpha}(t) d t+\lambda \int_{0}^{\omega} e(t) x(t) d t .
\end{aligned}
$$

Substituting $\int_{0}^{\omega} x^{\prime \prime}(t) x^{\prime}(t) d t=-\int_{0}^{\omega}\left|x^{\prime}(t)\right|^{2} d t$ and $\int_{0}^{T} f(x(t)) x(t) x^{\prime}(t) d t=0$ into Eq. (2.5), applying the Hölder inequality, we have

$$
\begin{aligned}
\int_{0}^{\omega}\left|x^{\prime}(t)\right|^{2} d t & =\lambda \int_{0}^{\omega} a(t)|x(t)|^{2} d t-\lambda \int_{0}^{\omega} b(t) x^{1-\alpha}(t) d t-\lambda \int_{0}^{\omega} e(t) x(t) d t \\
& \leq \int_{0}^{\omega}|a(t)||x(t)|^{2} d t+\int_{0}^{\omega}|e(t)||x(t)| d t \\
& \leq\|a\| \int_{0}^{\omega}|x(t)|^{2} d t+\|e\| \omega^{\frac{1}{2}}\left(\int_{0}^{T}\left|x^{\prime}(t)\right|^{2} d t\right)^{\frac{1}{2}}
\end{aligned}
$$

since $b(t)>0$ and $x(t)>0$. Define $u(t):=x(t+\xi)-x(\xi)$, where $\xi$ is as in Eq. (2.4), then $u(0)=u(\omega)=0$. Using Eq. (2.4), Lemma 2.1 and the Minkowski inequality, we deduce

$$
\begin{aligned}
\left(\int_{0}^{\omega}|x(t)|^{2} d t\right)^{\frac{1}{2}} & =\left(\int_{0}^{\omega}|u(t)+x(\xi)|^{2} d t\right)^{\frac{1}{2}} \\
& \leq\left(\int_{0}^{\omega}|u(t)| d t\right)^{\frac{1}{2}}+\left(\int_{0}^{\omega}|x(\xi)| d t\right)^{\frac{1}{2}} \\
& \leq \frac{\omega}{\sqrt{S(2)}}\left(\int_{0}^{\omega}\left|u^{\prime}(t)\right|^{2} d t\right)^{\frac{1}{2}}+\left(\sigma^{*}\right)^{\frac{1}{1+\alpha}} \omega^{\frac{1}{2}} \\
& \leq \frac{\omega}{\pi}\left(\int_{0}^{\omega}\left|x^{\prime}(t)\right|^{2} d t\right)^{\frac{1}{2}}+\left(\sigma^{*}\right)^{\frac{1}{1+\alpha}} \omega^{\frac{1}{2}}
\end{aligned}
$$

since $u^{\prime}(t)=x^{\prime}(t)$ and $S(2)=\pi^{2}$ from Remark 2.1. Substituting Eqs. (2.7) into (2.6), we arrive at

$$
\begin{aligned}
\int_{0}^{\omega}\left|x^{\prime}(t)\right|^{2} d t \leq & \|a\|\left(\frac{\omega}{\pi}\left(\int_{0}^{\omega}\left|x^{\prime}(t)\right|^{2} d t\right)^{\frac{1}{2}}+\left(\sigma^{*}\right)^{\frac{1}{1+\alpha}} \omega^{\frac{1}{2}}\right)^{2} \\
& +\|e\| \omega^{\frac{1}{2}} \frac{\omega}{\pi}\left(\left(\int_{0}^{\omega}\left|x^{\prime}(t)\right|^{2} d t\right)^{\frac{1}{2}}+\left(\sigma^{*}\right)^{\frac{1}{1+\alpha}} \omega^{\frac{1}{2}}\right) \\
= & \|a\| \frac{\omega^{2}}{\pi^{2}} \int_{0}^{\omega}\left|x^{\prime}(t)\right|^{2} d t+\frac{\omega^{\frac{3}{2}}}{\pi}\left(2\|a\|\left(\sigma^{*}\right)^{\frac{1}{1+\alpha}}+\|e\|\right)\left(\int_{0}^{\omega}\left|x^{\prime}(t)\right|^{2} d t\right)^{\frac{1}{2}} \\
& +\left(\|a\|\left(\sigma^{*}\right)^{\frac{2}{1+\alpha}}+\|e\|\left(\sigma^{*}\right)^{\frac{1}{1+\alpha}}\right) \omega \\
= & \|a\| \frac{\omega^{2}}{\pi^{2}} \int_{0}^{\omega}\left|x^{\prime}(t)\right|^{2} d t+N_{1}\left(\int_{0}^{\omega}\left|x^{\prime}(t)\right|^{2} d t\right)^{\frac{1}{2}}+N_{2}
\end{aligned}
$$


where $N_{1}:=\frac{\omega^{\frac{3}{2}}}{\pi}\left(2\|a\|\left(\sigma^{*}\right)^{\frac{1}{1+\alpha}}+\|e\|\right)$ and $N_{2}:=\left(\|a\|\left(\sigma^{*}\right)^{\frac{2}{1+\alpha}}+\|e\|\left(\sigma^{*}\right)^{\frac{1}{1+\alpha}}\right) \omega$. From condition $\left(H_{2}\right)$, we see that

$$
\left(1-\|a\| \frac{\omega^{2}}{\pi^{2}}\right) \int_{0}^{\omega}\left|x^{\prime}(t)\right|^{2} d t \leq N_{1}\left(\int_{0}^{\omega}\left|x^{\prime}(t)\right|^{2} d t\right)^{\frac{1}{2}}+N_{2}
$$

It is clear that

$$
\int_{0}^{\omega}\left|x^{\prime}(t)\right|^{2} d t \leq \frac{\pi^{2} N_{1}}{\pi^{2}-\|a\| \omega^{2}}\left(\int_{0}^{\omega}\left|x^{\prime}(t)\right|^{2} d t\right)^{\frac{1}{2}}+\frac{\pi^{2} N_{2}}{\pi^{2}-\|a\| \omega^{2}} .
$$

Furthermore, we obtain

$$
\left(\left(\int^{\omega}\left|x^{\prime}(t)\right|^{2} d t\right)^{\frac{1}{2}}-\frac{\pi^{2} N_{1}}{2 \pi^{2}-2\|a\| \omega^{2}}\right)^{2} \leq \frac{\pi^{2} N_{2}}{\pi^{2}-\|a\| \omega^{2}}+\frac{\pi^{4} N_{1}^{2}}{\left(2 \pi^{2}-2\|a\| \omega^{2}\right)^{2}} .
$$

Therefore, the above inequality implies

$$
\begin{aligned}
& \left(\int_{0}^{\omega}\left|x^{\prime}(t)\right|^{2} d t\right)^{\frac{1}{2}} \\
& \leq\left(\frac{\pi^{2} N_{2}}{\pi^{2}-\|a\| \omega^{2}}+\frac{\pi^{4} N_{1}^{2}}{\left(2 \pi^{2}-2\|a\| \omega^{2}\right)^{2}}\right)^{\frac{1}{2}}+\frac{\pi^{2} N_{1}}{2 \pi^{2}-2\|a\| \omega^{2}} \\
& =\left(\frac{\pi^{2} \omega\left(\| a \| \left(\sigma^{*} \frac{2}{1+\alpha}+\|e\|\left(\sigma^{*} \frac{1}{1+\alpha}\right)\right.\right.}{\pi^{2}-\|a\| \omega^{2}}+\frac{\pi^{2} \omega^{3}\left(2\|a\|\left(\sigma^{*}\right) \frac{1}{1+\alpha}+\|e\|\right)^{2}}{\left(2 \pi^{2}-2\|a\| \omega^{2}\right)^{2}}\right)^{\frac{1}{2}} \\
& \quad+\frac{\pi \omega^{\frac{3}{2}}\left(2\|a\|\left(\sigma^{*}\right) \frac{1}{1+\alpha}+\|e\|\right)}{2 \pi^{2}-2\|a\| \omega^{2}} \\
& \leq\left(\frac{\pi^{2} \omega\left(\|a\|\left(\sigma^{*}\right)^{\frac{2}{1+\alpha}}+\|e\|\left(\sigma^{*}\right)^{\frac{1}{1+\alpha}}\right)}{\pi^{2}-\|a\| \omega^{2}}\right)^{\frac{1}{2}}+\frac{\pi \omega^{\frac{3}{2}}\left(2\|a\|\left(\sigma^{*}\right) \frac{1}{1+\alpha}+\|e\|\right)}{\pi^{2}-\|a\| \omega^{2}}:=M_{1}^{\prime}
\end{aligned}
$$

where the lase inequality holds because of a classical inequality, i.e.,

$$
(a+b)^{k} \leq a^{k}+b^{k}, \quad \text { for } k \in(0,1), a, b \in(0,+\infty) .
$$

From Eqs. (2.4), (2.8) and the Hölder inequality, we deduce

$$
\begin{aligned}
x(t) & =\frac{1}{2}(x(t)+x(t-\omega)) \\
& =\frac{1}{2}\left(x(\xi)+\int_{\xi}^{t} x^{\prime}(s) d s+x(\xi)-\int_{t-\omega}^{\xi} x^{\prime}(s) d s\right) \\
& \leq x(\xi)+\frac{1}{2}\left(\int_{\xi}^{t}\left|x^{\prime}(s)\right| d s+\int_{t-\omega}^{\xi}\left|x^{\prime}(s)\right| d s\right) \\
& =x(\xi)+\frac{1}{2} \int_{t-\omega}^{t}\left|x^{\prime}(s)\right| d s \\
& \leq\left(\sigma^{*}\right)^{\frac{1}{1+\alpha}}+\frac{1}{2} \int_{0}^{\omega}\left|x^{\prime}(t)\right| d t
\end{aligned}
$$




$$
\begin{aligned}
\leq & \left(\sigma^{*}\right)^{\frac{1}{1+\alpha}}+\frac{\omega^{\frac{1}{2}}}{2}\left(\int_{0}^{\omega}\left|x^{\prime}(t)\right|^{2} d t\right)^{\frac{1}{2}} \\
\leq & \left(\sigma^{*}\right)^{\frac{1}{1+\alpha}}+\frac{\omega^{\frac{1}{2}}}{2}\left(\frac{\pi^{2} \omega\left(\|a\|\left(\sigma^{*}\right)^{\frac{2}{1+\alpha}}+\|e\|\left(\sigma^{*}\right)^{\frac{1}{1+\alpha}}\right)}{\pi^{2}-\|a\| \omega^{2}}\right)^{\frac{1}{2}} \\
& +\frac{\pi \omega^{2}\left(2\|a\|\left(\sigma^{*}\right)^{\frac{1}{1+\alpha}}+\|e\|\right)}{2\left(\pi^{2}-\|a\| \omega^{2}\right)}:=M_{1} .
\end{aligned}
$$

On the other hand, from Eqs. (2.4), (2.8) and (2.9), we get

$$
\begin{aligned}
x(t) & =x(\xi)+\frac{1}{2} \int_{t-\omega}^{t} x^{\prime}(s) d s \\
\geq & \left(\sigma_{*}\right)^{\frac{1}{1+\alpha}}-\frac{1}{2}\left(\int_{\xi}^{t}\left|x^{\prime}(s)\right| d s+\int_{t-\omega}^{\xi}\left|x^{\prime}(s)\right| d s\right) \\
\geq & \left(\sigma_{*}\right)^{\frac{1}{1+\alpha}}-\frac{1}{2} \int_{0}^{\omega}\left|x^{\prime}(t)\right| d s \\
\geq & \left(\sigma_{*}\right)^{\frac{1}{1+\alpha}}-\frac{\omega^{\frac{1}{2}}}{2}\left(\int_{0}^{\omega}\left|x^{\prime}(t)\right|^{2} d t\right)^{\frac{1}{2}} \\
\geq & \left(\sigma_{*}\right)^{\frac{1}{1+\alpha}}-\frac{\omega^{\frac{1}{2}}}{2}\left(\frac{\pi^{2} \omega\left(\|a\|\left(\sigma^{*} \frac{2}{1+\alpha}+\|e\|\left(\sigma^{*}\right)^{\frac{1}{1+\alpha}}\right)\right.}{\pi^{2}-\|a\| \omega^{2}}\right)^{\frac{1}{2}} \\
& -\frac{\pi \omega^{2}\left(2\|a\|\left(\sigma^{*}\right) \frac{1}{1+\alpha}+\|e\|\right)}{2\left(\pi^{2}-\|a\| \omega^{2}\right)}:=M_{2}>0,
\end{aligned}
$$

since $\sigma_{*}>\left(\frac{\omega^{\frac{1}{2}}}{2}\left(\frac{\pi^{2} \omega\left(\|a\|\left(\sigma^{*}\right) \frac{2}{1+\alpha}+\|e\|\left(\sigma^{*}\right) \frac{1}{1+\alpha}\right)}{\pi^{2}-\|a\| \omega^{2}}\right)^{\frac{1}{2}}+\frac{\pi \omega^{2}\left(2\|a\|\left(\sigma^{*}\right) \frac{1}{1+\alpha}+\|e\|\right)}{2\left(\pi^{2}-\|a\| \omega^{2}\right)}\right)^{1+\alpha}$ from condition $\left(H_{3}\right)$.

Next, we are going to obtain a uniform bound on $x^{\prime}(t)$. In fact, in view of $x(0)=x(\omega)$, there exists a point $t_{1} \in(0, \omega)$ such that $x^{\prime}\left(t_{1}\right)=0$, from Eqs. (2.2), (2.9) and (2.10), it is easy to see that

$$
\begin{aligned}
\left\|x^{\prime}\right\| & =\max _{t \in[0, \omega]}\left\{\left|x^{\prime}(t)\right|\right\} \\
& =\max _{t \in\left[t_{1}, t_{1}+\omega\right]}\left\{\left|\int_{t_{1}}^{t}\left(x^{\prime \prime}(s)\right) d s\right|\right\} \\
& \leq \int_{0}^{\omega}|f(x(t))|\left|x^{\prime}(t)\right| d t+\int_{0}^{\omega}|a(t)||x(t)| d t+\int_{0}^{\omega}\left|\frac{b(t)}{x^{\alpha}(t)}\right| d t+\int_{0}^{\omega}|e(t)| d t \\
& \leq\left|f_{M_{1}}\right| M_{1}^{\prime} \omega^{\frac{1}{2}}+\|a\| M_{1} \omega+\frac{\|b\| \omega}{M_{2}^{\alpha}}+\|e\| \omega:=M_{3},
\end{aligned}
$$

where $\left|f_{M_{1}}\right|:=\max _{M_{2} \leq x \leq M_{1}}|f(x)|$.

Having in mind Eqs. (2.9), (2.10) and (2.11), we define

$$
\Omega:=\left\{x \in X: E_{1}<x(t)<E_{2} \text { and }\left\|x^{\prime}\right\|<E_{3} \forall t \in \mathbb{R}\right\},
$$

where $X:=\{x \in C(\mathbb{R}, \mathbb{R}): x(0) \equiv x(\omega), \forall t \in \mathbb{R}\}, 0<E_{1}<M_{2}, E_{2}>M_{1}$ and $E_{3}>M_{3}$. Then the conditions (i) and (ii) of Lemma 2.2 are satisfied. From Eqs. (2.4), (2.9) and (2.10), we have

$$
\int_{0}^{\omega}\left(a(t) E_{1}-\frac{b(t)}{E_{1}^{\alpha}}\right) d t \cdot \int_{0}^{\omega}\left(a(t) E_{2}-\frac{b(t)}{E_{2}^{\alpha}}\right) d t<0 .
$$


Therefore, applying 2.2, we see that Eq. (1.1) has at least one positive periodic solution $x$ with

$$
\begin{array}{r}
x \in\left(\left(\sigma_{*}\right)^{\frac{1}{1+\alpha}}-\frac{\omega^{\frac{1}{2}}}{2}\left(\frac{\pi^{2} \omega\left(\|a\|\left(\sigma^{*}\right)^{\frac{2}{1+\alpha}}+\|e\|\left(\sigma^{*}\right)^{\frac{1}{1+\alpha}}\right)}{\pi^{2}-\|a\| \omega^{2}}\right)^{\frac{1}{2}}-\frac{\pi \omega^{2}\left(2\|a\|\left(\sigma^{*}\right)^{\frac{1}{1+\alpha}}+\|e\|\right)}{2\left(\pi^{2}-\|a\| \omega^{2}\right)},\right. \\
\left.\left(\sigma^{*}\right)^{\frac{1}{1+\alpha}}+\frac{\omega^{\frac{1}{2}}}{2}\left(\frac{\pi^{2} \omega\left(\|a\|\left(\sigma^{*}\right)^{\frac{2}{1+\alpha}}+\|e\|\left(\sigma^{*}\right)^{\frac{1}{1+\alpha}}\right)}{\pi^{2}-\|a\| \omega^{2}}\right)^{\frac{1}{2}}+\frac{\pi \omega^{2}\left(2\|a\|\left(\sigma^{*}\right)^{\frac{1}{1+\alpha}}+\|e\|\right)}{2\left(\pi^{2}-\|a\| \omega^{2}\right)}\right) .
\end{array}
$$

Next, we address the condition on the existence of a periodic solution for Eq. (1.1) with strong singularity.

Proof of Theorem 1.2 Similar to the proof of Theorem 1.1, from (2.4) and condition $\left(H_{4}\right)$, we know that there exists a point $\zeta \in(0, \omega)$ such that

$$
\left(\eta_{*}\right)^{\frac{1}{1+\alpha}} \leq x(\zeta) \leq\left(\eta^{*}\right)^{\frac{1}{1+\alpha}}
$$

where $\eta^{*}:=\frac{b}{\min _{t \in[0, \omega]} a(t)}, \eta_{*}:=\frac{b}{\max _{t \in[0, \omega]} a(t)}$. From Eq. (2.9), we get

$$
x(t) \leq M_{1} .
$$

Next, we claim that there exist two positive constants $M_{2}^{*}$ and $M_{3}^{*}$ such that

$$
x(t) \geq M_{2}^{*}, \quad \text { and } \quad\left\|x^{\prime}\right\| \leq \lambda M_{3}^{*}, \quad \lambda \in(0,1) .
$$

In fact, we first consider $\int_{0}^{\omega}\left|\frac{b}{x^{\alpha}(t)}\right| d t$ from condition $\left(H_{4}\right)$. Since $b>0$ and $x(t)>0$, from Eqs. (2.3) and (2.9), we obtain

$$
\begin{aligned}
\int_{0}^{\omega}\left|\frac{b}{x^{\alpha}(t)}\right| d t & =\int_{0}^{\omega} \frac{b}{x^{\alpha}(t)} d t \\
& =\int_{0}^{\omega} a(t) x(t) d t \\
& \leq\|a\| M_{1} \omega .
\end{aligned}
$$

Afterwards, from Eqs. (2.9), (2.11) and (2.13), we have

$$
\begin{aligned}
\left\|x^{\prime}\right\| & \leq \lambda\left(\int_{0}^{\omega}|f(x(t))|\left|x^{\prime}(t)\right| d t+\|a\| \int_{0}^{\omega}|x(t)| d t+\int_{0}^{\omega}\left|\frac{b}{x^{\alpha}(t)}\right| d t+\int_{0}^{\omega}|e(t)| d t\right) \\
& \leq \lambda\left(\left|f_{M_{1}}\right| M_{1}^{\prime} \omega^{\frac{1}{2}}+2\|a\| M_{1} \omega+\|e\| \omega\right):=\lambda M_{3}^{*} .
\end{aligned}
$$

On the other hand, multiplying both sides of Eq. (2.2) by $x^{\prime}(t)$ and integrating on $[\zeta, t]$, where $x(\zeta) \geq\left(\sigma_{*}\right)^{\frac{1}{1+\alpha}}$ is as in Eq. (2.12), we see that

$$
\begin{aligned}
& \int_{\zeta}^{t} x^{\prime \prime}(s) x^{\prime}(s) d s+\lambda \int_{\zeta}^{t} f(x(s))\left|x^{\prime}(s)\right|^{2} d s+\lambda \int_{\zeta}^{t} a(s) x(s) x^{\prime}(s) d s \\
& =\lambda \int_{\zeta}^{t} \frac{b x^{\prime}(s)}{x^{\alpha}(s)} d s+\lambda \int_{\zeta}^{t} e(s) x^{\prime}(s) d s .
\end{aligned}
$$


Furthermore, from Eq. (2.9) and (2.14), it is clear that

$$
\begin{aligned}
\lambda b\left|\int_{x(\zeta)}^{x(t)} \frac{d v}{v^{\alpha}}\right|= & \lambda\left|\int_{\zeta}^{t} \frac{b x^{\prime}(s)}{x^{\alpha}(s)} d s\right| \\
= & \left.\left|\int_{\zeta}^{t} x^{\prime \prime}(s) x^{\prime}(s) d s+\lambda \int_{\zeta}^{t} f(x(s))\right| x^{\prime}(s)\right|^{2} d s \\
& +\lambda \int_{\zeta}^{t} a(s) x(s) x^{\prime}(s) d s-\lambda \int_{\zeta}^{t} e(s) x^{\prime}(s) d s \mid \\
\leq & \frac{1}{2}\left(x^{\prime 2}(t)-x^{\prime 2}(\zeta)\right)+\lambda \int_{0}^{\omega}|f(x(s))|\left|x^{\prime}(s)\right|^{2} d s \\
& +\lambda \int_{0}^{\omega}|a(s)|\left|x^{\prime}(s)\right|^{2} d s+\lambda \int_{0}^{\omega}|e(s)|\left|x^{\prime}(s)\right| d s \\
\leq & \lambda^{2}\left(M_{3}^{*}\right)^{2}+\lambda\left|f_{M_{1}}\right| M_{3}^{*} \omega+\lambda\|a\| M_{1} M_{3}^{*} \omega+\lambda\|e\| M_{3}^{*} \omega
\end{aligned}
$$

since $b>0$. Therefore, the above inequality implies

$$
\left|\int_{x(\zeta)}^{x(t)} \frac{d v}{v^{\alpha}}\right| \leq \frac{M_{3}^{*}}{b}\left(M_{3}^{*}+\left|f_{M_{1}}\right| M_{3}^{*} \omega+\|a\| M_{1} \omega+\|e\| \omega\right):=M_{2}^{\prime}
$$

Since $\alpha \geq 1$, we get

$$
\left|\lim _{x \rightarrow 0^{+}} \int_{x}^{1} \frac{d v}{v^{\alpha}}\right|=\frac{1}{1-\alpha}+\frac{1}{\alpha-1} \lim _{x \rightarrow 0^{+}} \frac{1}{x^{\alpha-1}}=+\infty
$$

From Eq. (2.16) and $x(\zeta) \geq\left(\eta_{*}\right)^{\frac{1}{1+\alpha}}$, there exists a constant $M_{2}^{*} \in\left(0,\left(\eta_{*}\right)^{\frac{1}{1+\alpha}}\right)$ such that

$$
\int_{M_{2}^{*}}^{\left(\eta_{*}\right)^{\frac{1}{1+\alpha}}} \frac{d \nu}{v^{\alpha}}>M_{2}^{\prime}
$$

Thus, if there is a point $\zeta_{1} \in[\zeta, t]$ such that $x\left(\zeta_{1}\right) \leq M_{2}^{*}$, then

$$
\left|\int_{x\left(\zeta_{1}\right)}^{x(\zeta)} \frac{d v}{v^{\alpha}}\right| \geq\left|\int_{M_{2}^{*}}^{\left(\eta_{*}^{*}\right)^{\frac{1}{1+\alpha}}} \frac{d v}{v^{\alpha}}\right|>M_{2}^{\prime}
$$

which contradicts (2.15). Therefore, we get $x(t)>M_{2}^{*}$ for all $t \in[\zeta, \omega]$. For the case $t \in$ $[0, \zeta]$, we can proceed similarly.

The proof is the same as Theorem 1.1.

Remark 2.2 It is worth mentioning that the method of Theorem 1.2 is no longer applicable to the proof of existence of a positive periodic solution for Eq. (1.1) with weak singularity (i.e. $0<\alpha<1$ ). Due to $0<\alpha<1$, we cannot get the result that Eq. (2.16) holds, so we do not deduce that Eqs. (2.15) and (2.18) are a contradiction.

Remark 2.3 In condition $\left(H_{4}\right)$, we require that $b(t) \equiv b$. Actually, if $b(t)$ is a continuous periodic function rather than a positive constant, the work on estimating a lower bound of a positive periodic solution for Eq. (1.1) is no longer applicable. In fact, due to $b(t)$ 
being a function and $b(t) \not \equiv$ constant, it is easy to verify that $\left|\int_{\zeta}^{t} \frac{b(s) x^{\prime}(s)}{x^{\alpha}(s)} d s\right| \neq|b|\left|\int_{x(\zeta)}^{x(t)} \frac{d v}{v^{\alpha}} d s\right|$. Therefore, we cannot get Eq. (2.15).

Finally, we illustrate our results with two numerical examples.

Example 2.1 Consider the following Liénard equation with weak singularity:

$$
x^{\prime \prime}+x^{3} x^{\prime}+\left(\frac{1}{4} \sin 8 \pi t+\frac{1}{2}\right) x=\frac{\sin ^{2} 4 \pi t+6}{x^{\frac{1}{2}}}+\cos 8 \pi t .
$$

It is clear that $T=\frac{1}{4}, \alpha=\frac{1}{2}, f(x)=x^{3}, a(t)=\frac{1}{4} \sin 8 \pi t+\frac{1}{2}, b(t)=\sin ^{2} 4 \pi t+6, e(t)=\cos 8 \pi t$, $\sigma_{*}=8, \sigma^{*}=28$, and $\|a\|=\frac{3}{4}<16 \pi^{2}$, conditions $\left(H_{1}\right)$ and $\left(H_{2}\right)$ are satisfied. Next, we consider condition $\left(H_{3}\right)$,

$$
\begin{aligned}
& \frac{\omega^{\frac{1}{2}}}{2}\left(\frac{\pi^{2} \omega\left(\| a \| \left(\sigma^{*} \frac{2}{1+\alpha}+\|e\|\left(\sigma^{*} \frac{1}{1+\alpha}\right)\right.\right.}{\pi^{2}-\|a\| \omega^{2}}\right)^{\frac{1}{2}}+\frac{\pi \omega^{2}\left(2\|a\|\left(\sigma^{*} \frac{1}{1+\alpha}+\|e\|\right)\right.}{2\left(\pi^{2}-\|a\| \omega^{2}\right)} \\
& \quad=\frac{1}{4} \times\left(\frac{\pi^{2} \times \frac{1}{4} \times\left(\frac{3}{4} \times(28)^{\frac{4}{3}}+1 \times(28)^{\frac{2}{3}}\right)}{\pi^{2}-\frac{3}{64}}\right)^{\frac{1}{2}}+\frac{\pi \times \frac{1}{4} \times\left(\frac{3}{2} \times(28)^{\frac{2}{3}}+1\right)}{2 \pi^{2}-\frac{3}{32}} \\
& \quad \approx \frac{1}{4} \times(18.3355)^{\frac{1}{2}}+0.594 \\
& \quad \approx 1.6668<4=\left(\sigma_{*}\right)^{\frac{1}{1+\alpha}} .
\end{aligned}
$$

Furthermore, we get

$$
\begin{aligned}
& \left(\sigma_{*}\right)^{\frac{1}{1+\alpha}}-\frac{\omega^{\frac{1}{2}}}{2}\left(\frac{\pi^{2} \omega\left(\|a\|\left(\sigma^{*}\right) \frac{2}{1+\alpha}+\|e\|\left(\sigma^{*} \frac{1}{1+\alpha}\right)\right.}{\pi^{2}-\|a\| \omega^{2}}\right)^{\frac{1}{2}}-\frac{\pi \omega^{2}\left(2\|a\|\left(\sigma^{*}\right)^{\frac{1}{1+\alpha}}+\|e\|\right)}{2\left(\pi^{2}-\|a\| \omega^{2}\right)} \\
& >4-1.6668=2.3332, \\
& \left(\sigma^{*}\right)^{\frac{1}{1+\alpha}}+\frac{\omega^{\frac{1}{2}}}{2}\left(\frac{\pi^{2} \omega\left(\|a\|\left(\sigma^{*}\right)^{\frac{2}{1+\alpha}}+\|e\|\left(\sigma^{*}\right)^{\frac{1}{1+\alpha}}\right)}{\pi^{2}-\|a\| \omega^{2}}\right)^{\frac{1}{2}}+\frac{\pi \omega^{2}\left(2\|a\|\left(\sigma^{*}\right)^{\frac{1}{1+\alpha}}+\|e\|\right)}{2\left(\pi^{2}-\|a\| \omega^{2}\right)} \\
& \quad<9.221+1.6668=10.8878 .
\end{aligned}
$$

Therefore, applying Theorem 1.1, we know that Eq. (2.19) has at least one positive $\frac{1}{4}$ periodic solution $x$ with $x \in(2.3332,10.8878)$. Moreover, using Matlab, we can find a positive periodic solution for this equation as shown in Fig. 1.

Example 2.2 Consider the following Liénard equation with strong singularity:

$$
x^{\prime \prime}+x^{2} x^{\prime}+\left(\sin ^{2} 2 t+\frac{1}{2}\right) x=\frac{5}{x^{4}}
$$

It is obvious that $T=\frac{\pi}{2}, a(t)=\sin ^{2} 2 t+\frac{1}{2}, b=5\|a\|=\frac{3}{2}<4, \alpha=4$, then conditions $\left(H_{1}\right)$, $\left(H_{2}\right)$ and $\left(H_{4}\right)$ are satisfied. Hence, applying Theorem 1.2, we see that Eq. (2.20) has at least one positive $\frac{\pi}{2}$-periodic solution. Moreover, using Matlab, we can find a positive periodic solution for this equation as shown in in Fig. 2. 

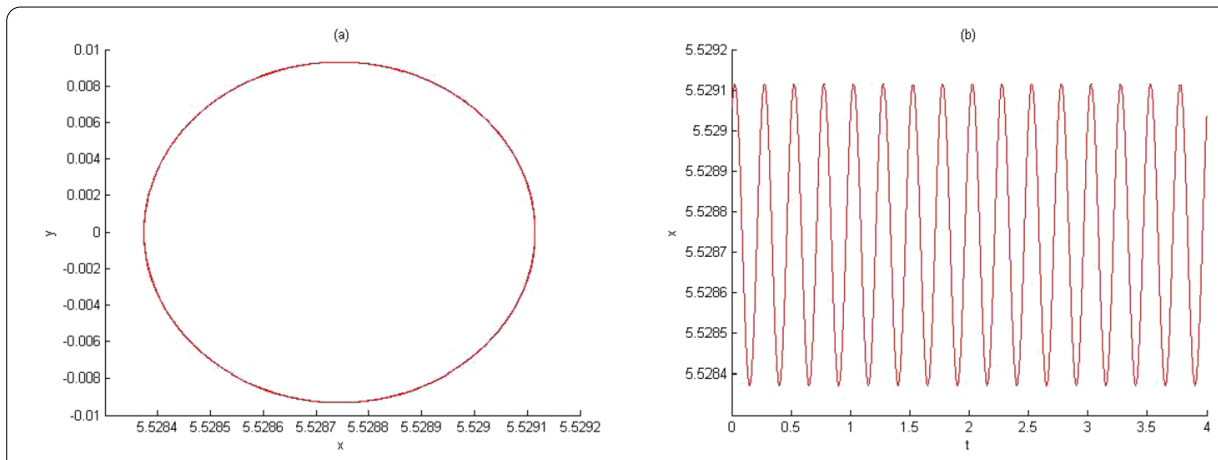

Figure 1 The first picture shows the system response in the $(x, y)$. The second shows the system response in the $(t, x)$ over the time interval of $0 \leq t \leq 50$. The initial conditions are $x_{0}=5.52907, y_{0}=0, t_{0}=0$

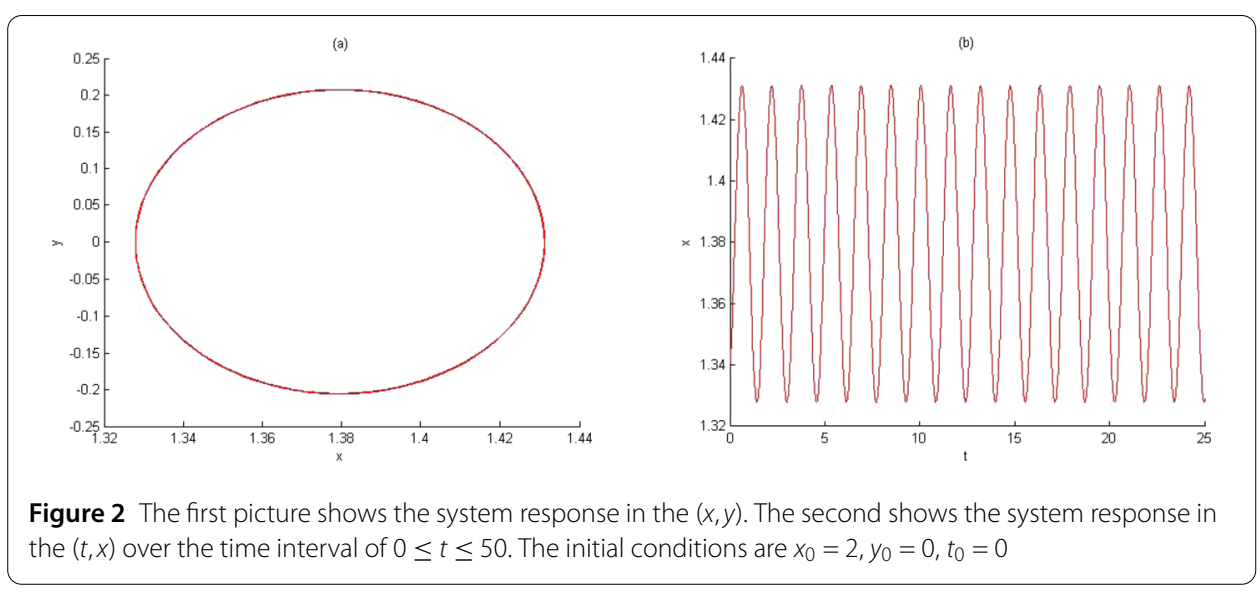

\section{Conclusions}

In this paper, applying an extension of the Manásevich-Mawhin continuation theorem, we investigate the existence of a periodic solution for Eq. (1.1), where the external force $e(t)$ may change sign, the singular term $\frac{b(t)}{x^{\alpha}}$ satisfies weak and strong singularities of repulsive type. Besides, we give the existence interval of periodic solution of Eq. (1.1). At last, two examples and numerical solutions (phase portraits and time portraits of periodic solutions of the example) are given to show applications of the theorem. The techniques used of this paper are quite different from that in $[5,8,10,17]$ and our results are more general than those in $[5,8,10,17]$ in two aspects. We first obtain the existence of a positive periodic solution for Eq. (1.1) with weak and strong singularities if the external force $e(t)$ may change sign. Secondly, we give the existence interval of positive periodic solution of Eq. (1.1).

\section{Acknowledgements}

YX and GXH are grateful to anonymous referees for their constructive comments and suggestions, which have greatly improved this paper.

\section{Funding}

This work was supported by Education Department of Henan Province project (No. 16B110006), Fundamental Research Funds for the Universities of Henan Province (NSFRF170302). 
Availability of data and materials

Not applicable.

\section{Ethics approval and consent to participate}

YX and GXH contributed to each part of this study equally and declare that they have no competing interests.

Competing interests

YX and GXH declare that they have no competing interests.

\section{Consent for publication}

YX and GXH read and approved the final version of the manuscript.

\section{Authors' contributions}

YX and GXH contributed equally and significantly in writing this article. Both authors read and approved the final manuscript.

\section{Author details}

${ }^{1}$ College of Computer Science and Technology, Henan Polytechnic University, Jiaozuo 454000, China. ${ }^{2}$ School of Mathematics and Information Science, Henan Polytechnic University, Jiaozuo 454000, China.

\section{Publisher's Note}

Springer Nature remains neutral with regard to jurisdictional claims in published maps and institutional affiliations.

Received: 6 June 2020 Accepted: 14 August 2020 Published online: 28 August 2020

\section{References}

1. Lazer, A., Solimini, S.: On periodic solutions of nonlinear differential equations with singularities. Proc. Am. Math. Soc. 99, 109-114(1987)

2. Cheng, Z., Yuan, Q.: Damped superlinear Duffing equation with strong singularity of repulsive type. J. Fixed Point Theory Appl. 22, 37 (2020)

3. Fonda, A., Manasevich, R., Zanolin, F.: Subharmonics solutions for some second order differential equations with singularities. SIAM J. Math. Anal. 24, 1294-1311 (1993)

4. Sun, X., Yu, P.: Exact bound on the number of zeros of Abelian integrals for two hyper-elliptic Hamiltonian systems of degree. J. Differ. Equ. 267, 7369-7384 (2019)

5. Cheng, Z., Ren, J.: Periodic solution for second order damped differential equations with attractive-repulsive singularities. Rocky Mt. J. Math. 48, 753-768 (2018)

6. Chu, J., Torres, P.: Applications of Schauder's fixed point theorem to singular differential equations. Bull. Lond. Math. Soc. 39, 653-660 (2007)

7. Liu, J., Cheng, Z., Wang, Y.: Positive periodic solution for second-order nonlinear differential equation with singularity of attractive type. J. Appl. Anal. Comput. 10, 1636-1650 (2020)

8. Torres, P.: Weak singularities may help periodic solutions to exist. J. Differ. Equ. 232, 277-284 (2007)

9. Cheng, Z., Li, F.: Weak and strong singularities for second-order nonlinear differential equations with a linear difference operator. J. Fixed Point Theory Appl. 21, 48 (2019)

10. Chu, J., Torres, P., Zhang, M.: Periodic solution of second order non-autonomous singular dynamical systems. J. Differ Equ. 239, 196-212 (2007)

11. Sun, X., Yu, P.: Periodic traveling waves in a generalized BBM equation with weak backward diffusion and dissipation terms. Discrete Contin. Dyn. Syst., Ser. B 24, 965-987 (2019)

12. Wang, Z:: Periodic solutions of Liénard equation with a singularity and a deviating argument. Nonlinear Anal., Real World Appl. 16, 227-234 (2014)

13. Xin, Y., Liu, H.: Singularities of attractive and repulsive type for p-Laplacian generalized Liénard equation. Adv. Differ. Equ. 2018, 471 (2018)

14. Xin, Y., Hu, G.: Periodic solution for prescribed mean curvature Rayleigh equation with a singularity. Adv. Differ. Equ. 2020, 269 (2020)

15. Zhang, M.: Periodic solutions of Liènard equation singular forces of repulsive type. J. Math. Anal. Appl. 203, 254-269 (1996)

16. Cheng, Z., Li, F.: Positive periodic solutions for a kind of second-order neutral differential equations with variable coefficient and delay. Mediterr. J. Math. 15, 134 (2018)

17. Wang, H.: Positive periodic solutions of singular systems with a parameter. J. Differ. Equ. 249, 2986-3002 (2010)

18. Hakl, R., Zamora, M.: Existence and uniqueness of a periodic solution to an indefinite attractive singular equation. Ann. Mat. Pura Appl. 195, 995-1009 (2016)

19. Hakl, R., Zamora, M.: Periodic solutions to second-order indefinite singular equations. J. Differ. Equ. 263, 451-469 (2017)

20. Mawhin, J.: Topological degree and boundary value problems for nonlinear differential equations. Topol. Methods Ordinary Differ. Equ. 1537, 74-142 (1993)

21. Talenti, G.: Best constant in Sobolev inequality. Ann. Mat. Pura Appl. 110, 353-372 (1976) 